九州大学学術情報リポジトリ

Kyushu University Institutional Repository

\title{
A P-Complete Language Describable with Iterated Shuffle
}

Shoudai, Takayoshi

Department of Control Engineering and Science Kyushu Institute of Technology

http://hdl. handle. net/2324/3152

出版情報 : RIFIS Technical Report. 46，1991-09-06. Research Institute of Fundamental Information Science, Kyushu University バージョン:

権利関係 : 


\title{
A P-Complete Language Describable with Iterated Shuffle
}

\author{
Takayoshi Shoudai \\ Department of Control Engineering and Science \\ Kyushu Institute of Technology \\ lizuka 820, Japan
}

September 6, 1991

\begin{abstract}
We show that a P-complete language can be described by using the shuffle operator, shuffle closure, union, concatenation, Kleene star and intersection on a finite alphabet.
\end{abstract}

\section{Introduction}

In this paper, we construct a P-complete language by using shuffle operator $\triangle$, iterated shuffle $\dagger$, union $U$, concatenation $\cdot$, Kleene star $*$ and intersection $\cap$ over a finite alphabet. The shuffle operator was introduced by [10] to describe the class of flow expressions. Formal properties of expressions with these operators have been extensively studied from various points in the literatures $[2,3,4,5,8,9,10,11]$.

It is known that the complexity of almost classes of languages can be increased by using the iterated shuffle operator. For example, there are two deterministic context-free languages $L_{1}$ and $L_{2}$ such that $L_{1} \triangle L_{2}$ is NP-complete [9]. Moreover, by allowing the synchronization mechanisms, any recursively enumerable set can be described $[1,3]$.

In $[2,11]$, by using the shuffle and iterated shuffle operators together with $\cup, \cdot, *, \cup$, an NPcomplete language is described. We employ the same set of operators to describe our P-complete language. In the proof of $\mathrm{P}$-completeness, the intersection operator plays an important role to make the language polynomial-time recognizable. However, we do not know whether the intersection operator is necessary to define a $\mathrm{P}$-complete language as in the case with NP-complete $[2,11]$.

Recently, P-complete problems have received considerable attentions since they do not seem to allow any efficient parallel algorithms [7]. This paper gives a $\mathrm{P}$-complete problem of a new kind, which is described by a single expression. 


\section{Preliminaries}

Let $\Sigma$ be a finite alphabet and $\Sigma^{*}$ be $\left\{a_{1} \cdots a_{n} \mid a_{i} \in \Sigma\right.$ for $i=1, \ldots, n$ and $\left.n \geq 0\right\}$. A subset of $\Sigma^{*}$ is called a language.

Definition 1 For languages $L, L_{1}$ and $L_{2}$, we define the shuffle operator $\triangle$, the iterated shuffle $\dagger$ and operators, $\cdot, *,+$ as follows:

(1) $L_{1} \triangle L_{2}=\left\{x_{1} y_{1} x_{2} y_{2} \cdots x_{m} y_{m} \mid x=x_{1} x_{2} \cdots x_{m} \in L_{1}, y=y_{1} y_{2} \cdots y_{m} \in L_{2}\right.$ and $x_{i}, y_{i} \in$ $\Sigma^{*}$ for $\left.i=1, \ldots, m\right\}$ (shuffle operator).

(2) $L^{\dagger}=\{\varepsilon\} \cup L \cup(L \triangle L) \cup(L \triangle L \triangle L) \cup \cdots$ (iterated shuffle).

(3) $L_{1} \cdot L_{2}=\left\{x y \mid x \in L_{1}\right.$ and $\left.y \in L_{2}\right\}$ (abbreviated to $L_{1} L_{2}$ ).

(4) $L^{*}=\{\varepsilon\} \cup L \cup(L \cdot L) \cup(L \cdot L \cdot L) \cdots$.

(5) $L^{+}=L \cdot L^{*}$.

We identify a language $\{w\}$ which consists of only one word with the $w$. Thus, we will denote $\{w\}^{*},\{w\}^{+},\{w\}^{\dagger}, \ldots$ by $w^{*}, w^{+}, w^{\dagger}$, respectively.

As the basis of our reduction, we use the circuit value problem (CVP) that was shown Pcomplete [6]. Our definition in this paper slightly different from one in [6].

\section{CIRCUIT VALUE PROBLEM (CVP)}

Instance: A circuit $C=\left(C_{1}, \ldots, C_{m}, C_{m+1}, \ldots, C_{n}\right)$, where each $C_{i}$ is either (i) $C_{i}=$ true or false $(1 \leq i \leq m)$, (ii) $C_{i}=\operatorname{NOR}\left(C_{j}, C_{k}\right)(m+1 \leq i \leq n$ and $j, k<i)$.

Problem: Decide whether the value of $C_{n}$ is true.

In later section, CVP represents the set of all circuits whose output is true.

Let $\Sigma$ be a finite alphabet, $v_{1}, v_{2}, \ldots, v_{m}$ be symbols where $v_{i} \in \Sigma$ for $i=1, \ldots, m$ and $w_{1}, w_{2}, \ldots, w_{m+1}$ be words on a alphabet $\Sigma-\left\{v_{1}, v_{2}, \ldots, v_{m}\right\}$. By using the iterated shuffle operation, a language $\left\{v_{1}^{n} v_{2}^{n} \cdots v_{m}^{n} \mid n \geq 1\right\}$ can be described as $\left(v_{1} v_{2} \cdots v_{m}\right)^{\dagger} \cap v_{1}{ }^{+} v_{2}+\cdots v_{m}+$. Moreover, we can represent $\left\{w_{1} v_{1}^{n} w_{2} v_{2}^{n} \cdots w_{m} v_{m}{ }^{n} w_{m+1} \mid n \geq 1\right\}$ as

$$
\left(w_{1} w_{2} \cdots w_{m+1} \triangle\left(v_{1} v_{2} \cdots v_{m}\right)^{\dagger}\right) \cap w_{1} v_{1}{ }^{+} w_{2} v_{2}+\cdots w_{m} v_{m}{ }^{+} w_{m+1} .
$$

We often use this form of languages to define a P-complete language. Whenever languages like these are defined in the next section, we will not describe the languages explicitly by using the shuffle operation and the iterated shuffle. 


\section{A P-complete language}

The main result in this paper is the following theorem.

Theorem 1 A P-complete language can be described with operators $\cdot, *, \cup, \cap, \triangle, \dagger$.

\subsection{Definition of the language}

We will describe a $\mathrm{P}$-complete language $\mathcal{L}$ with the alphabet $\Sigma=\{0,1, a, b, c, d, u, v, x, y\}$. This language is defined stepwize.

At first, a language $L$ is defined as follows:

$$
\begin{aligned}
L_{a} & =a^{+} 0 \cup a^{+} 1=\left\{a^{i} \beta \mid i \geq 1 \text { and } \beta \in\{0,1\}\right\} . \\
L_{b b a} & =\left(b^{+} 1 b^{+} 1 a^{+} 0\right) \cup\left(b^{+} 0 b^{+} 1 a^{+} 1\right) \cup\left(b^{+} 1 b^{+} 0 a^{+} 1\right) \cup\left(b^{+} 0 b^{+} 0 a^{+} 1\right) \\
& =\left\{b^{j} \beta^{\prime} b^{k} \beta^{\prime \prime} a^{i} \beta \mid i, j, k \geq 1 \text { and }\left(\beta^{\prime}, \beta^{\prime \prime}, \beta\right) \in\{(1,1,0),(0,1,1),(1,0,1),(0,0,1)\}\right\} \\
L_{b} & =b^{+} 1=\left\{b^{i} 1 \mid i \geq 1\right\} . \\
\hline L & =c L_{a}^{+} L_{b b a}{ }^{+} L_{b} .
\end{aligned}
$$

The following language $T$ (resp. $F$ ) is used for a distribution of true (resp. false) value.

$$
\begin{aligned}
T_{x} & =\left\{1 d x^{i} u^{i} \mid i \geq 1\right\}, \quad T_{y}=\left\{1 y^{i} v^{i} \mid i \geq 1\right\} . \\
T_{x y} & =\left\{1 d x^{i} u^{i} 1 y^{i} v^{i} \mid i \geq 1\right\}, \quad T_{y y}=\left\{1 y^{i} v^{i} 1 y^{i} v^{i} \mid i \geq 1\right\} . \\
& \\
T_{\text {odd }} & =T_{x y} T_{y y}{ }^{*} T_{y} \cap T_{x} T_{y y}{ }^{*}=\left\{1 d x^{i} u^{i}\left(1 y^{i} v^{i}\right)^{j} \mid i \geq 1, j \geq 1 \text { and } j \text { is odd. }\right\} . \\
T_{\text {even }} & =T_{x} T_{y y}{ }^{*} T_{y} \cap T_{x y} T_{y y}{ }^{*}=\left\{1 d x^{i} u^{i}\left(1 y^{i} v^{i}\right)^{j} \mid i \geq 1, j \geq 1 \text { and } j \text { is even. }\right\} . \\
& \\
\hline T & =T_{x} \cup T_{\text {odd }} \cup T_{\text {even }}=\left\{1 d x^{i} u^{i}\left(1 y^{i} v^{i}\right)^{j} \mid i \geq 1 \text { and } j \geq 0\right\} .
\end{aligned}
$$

$F$ is defined in a similar way. We use a symbol 0 instead of 1 which is used to construct the language $T$.

$$
F=\left\{0 d x^{i} u^{i}\left(0 y^{i} v^{i}\right)^{j} \mid i \geq 1 \text { and } j \geq 0\right\}
$$

Subwords $1 y^{i} v^{i}\left(\right.$ resp. $\left.0 y^{i} v^{i}\right)$ of a word in $T$ (resp. $F$ ) are combinated with $b^{i} 0$ (resp. $b^{i} 1$ ) of words in $L$ and decides the value of the $i$ th variable. These three languages $L, T$ and $F$ are combinated with each other by using the shuffle operation and the iterated shuffle. 


$$
\mathcal{J}=L \triangle(T \cup F)^{\dagger}
$$

A language $\mathcal{K}$ is used for our language to become polynomial time decidable. We construct the language $\mathcal{K}$ stepwize as follows:

$$
\begin{aligned}
& A_{11}=\left\{a^{i} 11 d x^{i} u^{i} \mid i \geq 1\right\} \\
& A_{00}=\left\{a^{i} 00 d x^{i} u^{i} \mid i \geq 1\right\} \\
& A_{01}=\left\{a^{i} 01 d x^{i} u^{i} \mid i \geq 1\right\}
\end{aligned}
$$

In a similar way, following languages are defined.

$$
\begin{aligned}
& B_{01}=\left\{b^{i} 01 y^{i} v^{i} \mid i \geq 1\right\} . \\
& B_{11}=\left\{b^{i} 11 y^{i} v^{i} \mid i \geq 1\right\} . \\
& M=\left(A_{11} \cup A_{00}\right)^{+}\left(B_{01} B_{01} A_{01}\right)^{+} B_{11} .
\end{aligned}
$$

The language $M$ has words whose subwords of the form $d x^{i} u^{i}$ corresponding to the $i$ th gate occurred more than two times a word. We want these subwords to be occured exactly one time a word.

$$
\begin{aligned}
N_{d} & =\left(d x u d x^{2} u^{2} \triangle(x u x u)^{\dagger}\right) \cap\left(d x^{+} u^{+} d x^{+} u^{+}\right)=\left\{d x^{i} u^{i} d x^{i+1} u^{n+1} \mid i \geq 1\right\} . \\
\hline N & =c\left(\left(d x u N_{d}^{*} \cap N_{d}{ }^{*} d x^{+} u^{+}\right) \cup\left(d x u N_{d}{ }^{*} d x^{+} u^{+} \cap N_{d}{ }^{*}\right)\right) \\
& =\left\{c d x u d x^{2} u^{2} \cdots d x^{i} u^{i} \mid i \geq 1\right\} .
\end{aligned}
$$

Then, we define a language $\mathcal{K}$ which will be used for allowing a language $\mathcal{J}$ to be in $\mathrm{P}$.

$$
\mathcal{K}=M \cap\left(N \triangle \Sigma^{\prime}\right), \text { where } \Sigma^{\prime}=\Sigma-\{d, u, x\} .
$$

Finally, we defined a language $\mathcal{L}$ as follows:

$$
\mathcal{L}=\mathcal{J} \cap \mathcal{K} .
$$

\subsection{Proof of the P-completeness}

Theorem 1 follows from a next lemma. 


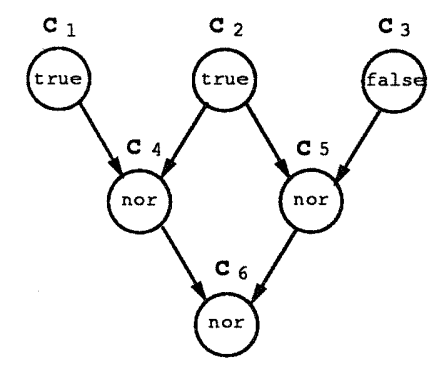

$$
\begin{aligned}
w= & a 11 d x u a^{2} 11 d x^{2} u^{2} a^{3} 00 d x^{3} u^{3} b 01 y v b^{2} 01 y^{2} v^{2} a^{4} 01 d x^{4} u^{4} \\
& b^{2} 01 y^{2} v^{2} b^{3} 01 y^{3} v^{3} a^{5} 01 d x^{5} u^{5} b^{4} 01 y^{4} v^{4} b^{5} 01 y^{5} v^{5} a^{6} 01 d x^{6} u^{6} b^{6} 11 y^{6} v^{6}
\end{aligned}
$$

Figure 1: This circuit is transformed to a word $w$.

Lemma $1 \mathcal{L}$ is log-space equivalent to CVP, i.e., $\mathcal{L}$ is log-space reducible from CVP and CVP is $\log$-space reducible from $\mathcal{L}$.

Proof. We will define a function $f$ from CVP to $\Sigma^{*}$. $f$ is a function which transform $C=$ $\left(C_{1}, \ldots, C_{n}\right) \in \mathrm{CVP}$ to $f(C)=\gamma w_{1} \cdots w_{n} w_{n+1} \in \Sigma^{*}$, where

$$
w_{i}= \begin{cases}a^{i} 11 d x^{i} u^{i} & \left(C_{i}=\text { true }\right) \\ a^{i} 00 d x^{i} u^{i} & \left(C_{i}=\text { false }\right) \\ b^{j} 01 y^{j} v^{j} b^{k} 01 y^{k} v^{k} a^{i} 01 d x^{i} u^{i} & \left(C_{i}=\operatorname{NOR}\left(C_{j}, C_{k}\right)\right) \\ b^{n} 11 y^{n} v^{n} & (i=n+1) .\end{cases}
$$

It is easy to see that this function is computable in log-space by using a deterministic Turing machine.

We show following two claims.

Claim 1. $f(C) \in \mathcal{L}$, for every $C \in \mathrm{CVP}$.

Proof. Let a word $w=c w_{1} \cdots w_{m} w_{m+1} \cdots w_{n} w_{n+1}$ be a transformed word from some $n$-gates instance $C=\left(C_{1}, \ldots, C_{m}, C_{m+1}, \ldots, C_{n}\right)$ where $C_{i}$ is an input gate for $1 \leq i \leq m$, an and gate for $m+1 \leq i \leq n$ and an output of this circuit is true. This instance has only one tuple of assignments of a boolean value (true or false) to each variables. We describe this assignment as $B=\left(\beta_{1}, \ldots, \beta_{n}\right)$ such that $\beta_{i}=1$ (resp. $\beta_{i}=0$ ) if $C_{i}=$ true (resp. $C_{i}=$ false) for $i=1, \ldots, n$.

According to $B=\left(\beta_{1}, \ldots, \beta_{n}\right)$, we divide $w_{i}$ into two words $w_{i}{ }^{\prime}$ and $w_{i}{ }^{\prime \prime}$.

(1) For $i=1, \ldots, m, w_{i}{ }^{\prime}=a^{i} \beta_{i}, w_{i}{ }^{\prime \prime}=\beta_{i} d x^{i} u^{i}$.

(2) For $i=m+1, \ldots, n, w_{i}{ }^{\prime}=b^{j} \bar{\beta}_{j} b^{k} \bar{\beta}_{k} a^{i} \bar{\beta}_{i}, w_{i}{ }^{\prime \prime}=\beta_{j} y^{j} v^{j} \beta_{k} y^{k} v^{k} \beta_{i} d x^{i} u^{i}$.

We note that since $C_{j}, C_{k}$ and $C_{i}$ are related with each other by an NOR gate, $w_{i}{ }^{\prime}$ is in $L_{b b a}$. 
(3) $w_{n+1}{ }^{\prime}=b^{n} 1, w_{n+1}{ }^{\prime \prime}=1 y^{n} v^{n}$.

It is easy to see that a word $w^{\prime}=c w_{1}{ }^{\prime} \cdots w_{n+1}{ }^{\prime}$ is in $L=L_{a}{ }^{+} L_{b b a}{ }^{+} L_{b}$.

On the other hand, since $w^{\prime \prime}=w_{1}{ }^{\prime \prime} \cdots w_{n+1}{ }^{\prime \prime}$ is constructed with subwords of the form $\beta_{i} d x^{i} u^{i}$ or $\beta_{i} y^{i} v^{i}$ and for each NOR gate, input gate nubers of this gate are always lower than a number of itself, we can describe the word $w^{\prime \prime} \in t_{1} \triangle t_{2} \triangle \cdots \Delta t_{n}$, where $t_{i}=\beta_{i} d x^{i} u^{i} \beta_{i} y^{i} v^{i} \cdots \beta_{i} y^{i} v^{i}$. Since $t_{i} \in$ $T$ or $F$, for $i=1, \ldots, n, f(C)=c w_{1} \cdots w_{m} w_{m+1} \cdots w_{n} w_{n+1} \in w^{\prime} \triangle t_{1} \triangle \cdots t_{n} \subset L \triangle(T \cup F)^{\dagger}=\mathcal{L}$.

Since every words $w$ of $\mathcal{L}$ is contained in $M, c$ is of the form $w=c w_{1} \cdots w_{m} w_{m+1} \cdots w_{n} w_{n+1}$, where, for $i=1, \ldots, n+1$,

$$
w_{i}= \begin{cases}a^{\ell_{i}} \beta_{i} \beta_{i} d x^{\ell_{i}} u^{\ell_{i}} & \left(1 \leq i \leq m, \beta_{i} \in\{0,1\}\right) \\ b^{\ell^{\prime}} 01 y^{\ell_{i}} v^{\ell_{i}} b^{\ell_{i}{ }^{\prime \prime}} 01 y^{\ell_{i}{ }^{\prime}} v^{\ell_{i}{ }^{\prime \prime}} a^{\ell_{i}} 01 d x^{\ell_{i}} u^{\ell_{i}} & (m+1 \leq i \leq n) \\ b^{\ell_{n+1}} 11 y^{\ell_{n+1}} v^{\ell_{n+1}} & (i=n+1)\end{cases}
$$

We transform a word $w \in \mathcal{L}$ to a circuit $C=\left(C_{1}, \ldots, C_{m}, C_{m+1}, \ldots, C_{n}\right)$ as follows:

(1) For $i=1, \ldots, m$, if $\beta_{i}=1$ then $C_{i}=$ true else $C_{i}=$ false.

(2) For $i=m+1, \ldots, n, C_{i}=\operatorname{NOR}\left(C_{j}, C_{k}\right)$ where $j=\ell_{i}^{\prime}$ and $k=\ell_{i}{ }^{\prime \prime}$.

It is easy to see that $g$ is well-defined function and this function is log-space computable.

Claim 2. $g(w) \in C V P$, for every $w \in \mathcal{L}$.

Proof. Since $w \in N, \ell_{i}=i$ for every $i=1, \ldots, n$. Moreover, since some parts of $w$ are constructed of words which are contained in $T$ or $F$, a subword $y^{i} v^{i}$ of $w$ is never occured before a subword $d x^{i} u^{i}$ of $w$. Therefore $j, k \leq i$.

Since $w \in L \triangle(T \cup F)^{\dagger}$ and $w$ includes $n$ subwords $d x u, d x^{2} u^{2}, \ldots, d x^{n} u^{n}$, there exist $n$ words $t_{1}, \ldots, t_{n}$ in $T \cup F$ which contribute a construction of $w$ by using the iterated shuffle. Without loss of generality, we assume that $t_{i}$ includes $x^{i} u^{i}$ as a subword.

We claim that for $i=1, \ldots, n, t_{i} \in T$ if and only if a value of $C_{i}$ is true. This is shown by the induction. For $i=1, \ldots, m$, if $\beta_{i}=1$, then $t_{i}$ must be in $T$. Thus, by definition of $g, C_{i}=$ true. For $i \geq m+1$, suppose that for $j, k<i$, this claim is true. We only discuss the case of $t_{j} \in T$ and $t_{k} \in T$. Other case is shown in a similar way. By the assumption, values of $C_{j}$ and $C_{k}$ is $t r u e$. We remove contributions of $t_{j}$ and $t_{k}$ from $w_{i}$. The remaining word is $b^{j} 0 b^{k} 0 a^{i} 01 d x^{i} u^{i}$. Moreover, $w_{i}$ must has a contribution from $L_{b b a}$. This contribution must be of the form $b^{+} 0 b^{+} 0 a^{+} 1$. Thus, the remaining word after removing this contribution is $0 d x^{i} u^{i}$. Therefore, $t_{i}$ must be in $F$. On the other hand, a value of $C_{i}=\operatorname{NOR}\left(C_{j}, C_{k}\right)$ is false. Thus, we hold this claim.

Since $t_{n}$ must be in $T$, a value of $C_{n}$ is true. Thus, $g(w) \in C V P$.

By the discussion above, we can say that $\mathcal{L}$ have a log-space reduction $f$ from CVP and CVP have a $\log$-space reduction $g$ (inverse of $f$ ) from $\mathcal{L}$. 


\section{References}

[1] T. Araki and N. Tokura, Flow languages equal recursively enumerable languages, Acta. Inform. 15 (1978) 209-217.

[2] T. Hayashi and S. Miyano, Flow expressions and complexity analysis, Reports of WGSF Meeting of Infomation Processing Society of Japan SF2-3 (1982) 1-10.

[3] M. Jantzen, The power of synchronizing operations on strings, Theoret. Comput. Sci. 14 (1981) $127-154$.

[4] M. Jantzen, Extending regular expressions with iterated shuffle, Theoret. Comput. Sci. 38 (1985) 223-247.

[5] J. Jedrzejowicz, On the enlargement of the class of regular languages by the shuffle closure,Inform. Proc. Lett. 16 (1983) 51-54.

[6] R.E. Ladner, The circuit value problem is log space complete for P, SIGACT News 7, 18-20.

[7] S. Miyano, S. Shiraishi and T. Shoudai, A list of P-complete problems, RIFIS-TR-CS-17, Research Institute of Fundamental Information Science, Kyushu University, 1989 (revised in December, 1990).

[8] M. Nivat, Behaviors of processes and synchronized systems of processes, Lecture note at Marktoberdopf NATO Summer School 1981.

[9] W.F. Ogden, W.E. Riddle and W.C. Rounds, Complexity of expressions allowing concurrency, Conference Record of the 5th Annual ACM Symposium on Principles of Programming Languages (1978) 185-194.

[10] A.C. Shaw, Software descriptions with flow expressions, IEEE Trans. Software Engrg. SE-4(3) (1978) 242-254.

[11] M.K. Warmuth and D. Haussler, On the complexity of iterated shuffle, J. Comput. Syst. Sci. 28 (1984) 345-358. 